\title{
Hyperspectral discrimination of tree species with different classifications using single- and multiple-endmember.
}

\begin{abstract}
Discrimination of tree species with different ages is performed in three classifications using hyperspectral data. The first classification is between Broadleaves and pines; the second classification is between Broadleaves, Corsican Pines, and Scots Pines, and the third classification is between six tree species including different ages of Corsican and Scots Pines. These three classifications are performed by having single- and multiple-endmember and considering five different spectral measure techniques (SMTs) in combination with reflectance spectra (ReflS), first and second derivative spectra. The result shows that using single-endmember, derivative spectra are not useful for a more challenging classification. This is further emphasized in multiple-endmember classification, where all SMTs perform better in ReflS rather than derivative in all classifications. Furthermore, using derivative spectra, discrimination accuracy become more dependent on the type of SMTs, especially in single-endmember. By employing multiple-endmember, the within-species variation is significantly reduced, thereby, the remaining challenge in discriminating tree species with different ages is only due to the between-species similarity. Overall, discrimination accuracies around 92.4, 76.8, and $71.5 \%$ are obtained using original reflectance and multipleendmember for the first, second, and third classification, which is around 14.3, 17, and 8.3\% higher than what were obtained in single-endmember classifications, respectively. Also, amongst the five SMTs, Euclidean distance (in both single- and multiple-endmember) and Jeffreys-Matusita distance (in single-endmember and derivative spectra) provided the highest discrimination accuracies in different classifications. Furthermore, when discrimination become more challenging from the first to second and third classification, the performance difference between different SMTs is increased from 1.4 to 3.8 and $7.3 \%$, respectively. The study shows high potential of multiple-endmember to be employed in remote sensing applications in the future for improving tree species discrimination accuracy.
\end{abstract}

Keyword: Remote sensing; End-members; Vegetation 